\title{
The quest for patient safety
}

\section{Gauri Raman Gangakhedkar}

Department of Anesthesiology, Seth Gordhandas Sunderdas Medical College and King Edward Memorial Hospital, Mumbai, India

Anesthesiologists have played a special role in improving patient safety. By analyzing factors that could potentially harm patients and developing strategies to manage them, they have raised the bar in measuring safety. In the last three decades, anesthesia-related mortality has dropped to $0.04-7$ per 10,000 anesthetics, representing a 10-fold decrease since the 1980s [1]. Human factors have been identified in $51 \%-77 \%$ of anesthesia-related deaths [1], thus indicating the need to strive for further improvement in the numbers and to ensure that errors are not repeated.

Further analysis of human factors revealed lack of experience or competence in $89 \%$ of the deaths and errors of judgment or analysis in $11 \%$ [1]. In their landmark paper, Kruger and Dunning [2] suggested that inexperienced or incompetent individuals do not possess the degree of metacognitive skills necessary for accurate self-assessment. They found that participants of their study scoring in the bottom-quartile were less capable of gauging the competence of both themselves and others as compared to their top-quartile counterparts. The clinical translation of this Dunning-Kruger effect is that unskilled trainees face a dual burden: they would continue to make mistakes and would not possess the skills to understand or correct their actions.

The same study also showed that on training the same inexperienced individuals, they not only performed better but also showed better self and peer assessment skills, thus proving the

Corresponding author: Gauri Raman Gangakhedkar, D.N.B. Anaesthesiology

Department of Anesthesiology, Seth Gordhandas Sunderdas Medical College and King Edward Memorial Hospital, Mumbai 400012, Maharashtra, India

Tel: 91-9096266328

Email: gauri2903@gmail.com

ORCID: https://orcid.org/0000-0001-6302-6804

Received: August 31, 2018.

Revised: September 13, 2018.

Accepted: October 4, 2018.

Korean J Anesthesiol 2019 June 72(3): 281-282

https://doi.org/10.4097/kja.d.18.00258 importance of incorporating patient safety programs in the formal clinical curriculum.

A systematic approach would be required to reduce errors occurring due to human factors. To begin with, the anesthesiology curriculum must be updated to address the lacunae of the current curriculum that usually consists of theoretical teaching and practical education on real patients. Owing to this, exposure to rare critical scenarios is limited, and in the case of occurrence of such events, neither the time nor the tools are available to train the anesthesia team during the course of the event. Simulation seems like an obvious answer in this regard, but its widespread applicability is limited by the high costs.

Furthermore, debriefing after critical incidents and regularly conducting problem-based learning discussions, using videos and manikins, could be a low-cost alternative to simulation in developing countries.

One of the most important concerns with implementing patient safety programs is training mentors to teach. Most patient safety training programs are designed to train individuals, not mentors [3]. The WHO Patient Safety Curriculum 2011 is a multi-professional guide that helps to train mentors to incorporate patient safety in the training program effectively [4]. Inclusion of such programs would arguably help in better training of clinicians and improve patient safety.

The clinical practice of anesthesia involves a dynamic work environment with rapidly changing requirements for anesthesiologists due to physiological changes, unforeseen surgical events, or other events requiring attention like shifting patients to post-anesthesia care units. Practice is typically team-based with dynamic roles that require adaptability and implicit coordination. Simulation studies have shown that teams performed better when members shared leadership and used explicit leadership in less standardized situations for example, sudden surgical bleed or anaphylactic shock and shared leadership in routine situations [5].

Mentors thus play an extremely important role in patient safety training. They lead by example, provide active leadership during a crisis, and possess the unique advantage of being able to train the unskilled and also recognize and train potential

(c) This is an open-access article distributed under the terms of the Creative Commons Attribution Non-Commercial License (http://creativecommons.org/ licenses/by-nc/4.0/), which permits unrestricted non-commercial use, distribution, and reproduction in any medium, provided the original work is properly cited. 
leaders.

Peer-led education is also becoming a popular method of education, but whether it is more effective than conventional education remains to be determined.

In conclusion, the quest for patient safety is endless, but a better understanding of human factors leading to errors, attempting a root cause analysis to eliminate them, and proper utilization of resources for patient safety training will definitely help to ensure safer anesthetic practices in the future.

\section{Conflicts of Interest}

No potential conflict of interest relevant to this article was reported.

\section{Author Contributions}

Gauri Raman Gangakhedkar (Conceptualization; Data curation; Investigation; Writing - original draft; Writing - review \& editing)

\section{References}

1. Haller G, Laroche T, Clergue F. Morbidity in anaesthesia: today and tomorrow. Best Pract Res Clin Anaesthesiol 2011; 25: 123-32.

2. Kruger J, Dunning D. Unskilled and unaware of it: how difficulties in recognizing one's own incompetence lead to inflated self-assessments. J Pers Soc Psychol 1999; 77: 1121-34.

3. Leotsakos A, Ardolino A, Cheung R, Zheng H, Barraclough B, Walton M. Educating future leaders in patient safety. J Multidiscip Healthc 2014; $7: 381-8$.

4. World Health Organization. Patient Safety Curriculum Guide: Multi-professional Edition [Serial on the Internet]. Geneva: WHO Press 2011 [cited 2018 August 31]. Available from http://www.who.int/patientsafety/education/mp_curriculum_guide/en/

5. Wacker J, Kolbe M. Leadership and teamwork in anesthesia-making use of human factors to improve clinical performance. Trends Anaesth Crit Care 2014; 4: 200-5. 